\title{
Fermionic Superfluidity with Imbalanced Spin Populations and the
}

\author{
Quantum Phase Transition to the Normal State \\ Martin W. Zwierlein * André Schirotzek, Christian H. Schunck, and Wolfgang Ketterle \\ Department of Physics, MIT-Harvard Center for \\ Ultracold Atoms, and Research Laboratory of Electronics, \\ MIT, Cambridge, MA 02139
}

(Dated: January 5, 2018)

\begin{abstract}
Whether it occurs in superconductors, helium-3 or inside a neutron star, fermionic superfluidity requires pairing of fermions, particles with half-integer spin. For an equal mixture of two states of fermions ("spin up" and "spin down"), pairing can be complete and the entire system will become superfluid. When the two populations of fermions are unequal, not every particle can find a partner. Will the system nevertheless stay superfluid? Here we study this intriguing question in an unequal mixture of strongly interacting ultracold fermionic atoms. The superfluid region vs population imbalance is mapped out by employing two complementary indicators: The presence or absence of vortices in a rotating mixture, as well as the fraction of condensed fermion pairs in the gas. Due to the strong interactions near a Feshbach resonance, the superfluid state is remarkably stable in response to population imbalance. The final breakdown of superfluidity marks a new quantum phase transition, the Pauli limit of superfluidity.
\end{abstract}


The study of superfluidity in an unequal mixture of two Fermi gases, or more generally unequal Fermi surfaces, is highly relevant to a wide area of physics. For example, this situation should occur in strongly degenerate quark matter in the core of a neutron star, where quarks of differing mass will belong to Fermi spheres of differing size. The ground state of such a system has been the subject of debate for decades [1, 2, 3, 4, 5] and experimental studies are highly desirable. However, the experimental realization of unbalanced Fermi seas in superconductors, charged fermionic superfluids, poses extreme difficulties: To reach imbalanced electron densities of spin up vs spin down electrons, a natural idea would be to apply a magnetic field. However, magnetic fields are either fully shielded from the superconductor via the Meissner effect, or they enter only in the form of quantized flux lines or vortices. Experiments trying to study mismatched Fermi surfaces therefore have to suppress these effects, as in experiments in heavy fermion superconductors [6, 7], 8] or quasi-two-dimensional organic superconductors [6]. In the neutral superfluid helium-3, one can mismatch the Fermi surfaces by a magnetic field and thus destroy inter-spin pairing. However, superfluidity persists due to (p-wave) pairing between equal spins 91 .

The recently discovered atomic fermionic superfluids [10, 11, 12, 13, 14, 15, 16, 17, 18, 19, 20, 21, 22] provide an exciting new possibility to explore unequal mixtures of fermions. Here, populations in two hyperfine states of the fermionic atom can be freely chosen. In addition, the (s-wave) interactions between two atoms in different states and hence the binding energy of atom pairs can be tuned. In equal mixtures of fermions, this tunability is being exploited to study the crossover from a Bose-Einstein Condensate (BEC) of molecules to a BardeenCooper-Schrieffer (BCS) superfluid of loosely bound pairs [13, 14, 15, 16, 19, 21, 22]. At zero temperature, this crossover is smooth [23, 24, 25], the system stays superfluid even for arbitrarily weak interaction and no phase transition occurs. In the case of unequal mixtures, the phase diagram is predicted to be much richer [26, 27, 28, 29, 31, 32]. In the molecular limit of tight binding, all fermions in the less populated spin state will pair up with atoms in the other state. The resulting molecular condensate will spatially coexist with the remaining Fermi sea of unpaired atoms. As the repulsive interaction between atoms and molecules is increased, the condensate will start to expel unpaired atoms, leading to a phase separation of the superfluid from the normal phase [26, 28, 29, 33, 34]. This picture is expected [26, 28, 29, 35] to extend into the BCS-limit of weakly bound pairs. Here, the pairing gap $\Delta$ prevents unpaired atoms from entering the BCS superfluid [35]. 
As the binding and hence the pairing gap is further reduced, $\Delta$ will eventually become small compared to the chemical potential difference $\delta \mu=\mu_{2}-\mu_{1}$ between the two spin states, allowing unpaired excess atoms to enter the superfluid region. Close to this point, superfluidity will cease to exist [30]. In the weakly interacting BCS-limit the pairing gap is exponentially small compared to the Fermi energy, hence an exponentially small population imbalance can destroy superfluidity.

This superfluid to normal transition is an example of a quantum phase transition, which occurs even at zero temperature, when all thermal fluctuations are frozen out and only quantum fluctuations prevail. It can also be driven by increasing the mismatch in chemical potentials between the two spin states to the critical value of $\delta \mu \approx \Delta$, inducing collapse into the normal state. In this context the phase transition is known as the Pauli or Clogston limit of superfluidity [1]. Its exact nature, whether there is one or several first-order and/or second-order transitions, however, is still the subject of current debate 66, 31, 32].

In this paper we map out the superfluid region as a function of population imbalance, interaction strength and temperature in an ultracold fermionic gas of ${ }^{6} \mathrm{Li}$ atoms. The only direct and unambiguous signature of superfluid flow in Fermi gases so far is the presence of vortices [22]. By studying unequal Fermi mixtures under rotation we establish superfluidity for a broad range in the population mismatch. Close to the breakdown of superfluidity, vortices are strongly damped and difficult to observe. Therefore, we map out the full regime of superfluidity by determining the fraction of condensed fermion pairs in a non-rotating cloud [14, 15].

The two experimental methods require slightly different procedures for imaging the pair condensate wavefunction after release from the trap. To extract the fraction of condensed vs uncondensed pairs, the condensate must separate well from the thermal cloud and should therefore remain small. For the detection of rotating clouds, the condensate should expand to a large size in order to magnify the vortices. In the following, we give the parameters used to determine the condensate fraction in parentheses after those used for vortex detection.

In the experiment, fermionic ${ }^{6} \mathrm{Li}$ atoms were sympathetically cooled to degeneracy by ${ }^{23} \mathrm{Na}$ atoms in a magnetic trap [36]. The ultracold cloud was subsequently loaded into an optical dipole trap (waist $w \approx 120 \mu \mathrm{m}$ ) at a maximum trap depth of about $8 \mu \mathrm{K}$. At a magnetic bias field of $875 \mathrm{G}$, a variable spin-mixture of the two lowest hyperfine states (labelled $|1\rangle$ and $|2\rangle)$ was created via a Landau-Zener sweep with variable sweep rate. Interactions between 
these two states are strongly enhanced around a $300 \mathrm{G}$ wide Feshbach resonance located at $834 \mathrm{G}$ [37]. At lower values of the magnetic bias field, two isolated fermions can bind into a stable molecule, while at higher values fermion pairs can only exist in the stabilizing presence of the surrounding gas. This tunability of the binding energy provides access to the BEC-BCS crossover physics. The spin mixture was evaporatively cooled further by lowering the trap depth to $1.6 \mu \mathrm{K}$ resulting in radial and axial trap frequencies of $\nu_{r}=110 \mathrm{~Hz}$ and $\nu_{a}=23 \mathrm{~Hz}$, respectively. At the same time, the magnetic field was ramped to $812 \mathrm{G}$ (818 $\mathrm{G}$ ), which is on the BEC-side of the resonance, but still in the regime of strong interaction. Here, $1 / k_{F} a=0.19(0.11)$, where $a$ is the scattering length and $k_{F}$ is defined as the Fermi momentum of a non-interacting, equal spin mixture. The rather moderate evaporation still leaves room for thermal molecules in an equal mixture, but was chosen to ensure efficient cooling of highly asymmetric mixtures, avoiding spilling of large Fermi clouds. It ensured that the total number of atoms $N=7 \cdot 10^{6}\left(N=2.310^{7}\right)$ was approximately constant and independent of the asymmetry between the two spin states.

For the vortex experiment, we set the spin mixture in rotation using two blue-detuned laser beams (wavelength $532 \mathrm{~nm}$ ) rotated symmetrically around the cloud at angular frequency $\Omega=2 \pi 70 \mathrm{~Hz}$ ) [22]. After $800 \mathrm{~ms}$ of stirring, the rotating cloud was left to equilibrate for several hundred ms.

Starting with either the rotating or the non-rotating cloud, we then varied the interaction strength between the two spin states in the gas by ramping the magnetic field in $100 \mathrm{~ms}$ (500 ms) to several values around the Feshbach resonance (for the condensate fraction experiment, the trap depth was simultaneously increased to $\left.4 \mu \mathrm{K}\left(\nu_{r}=192 \mathrm{~Hz}\right)\right)$. After $50 \mathrm{~ms}(100 \mathrm{~ms})$ of hold time, an image of the cloud was taken following the procedure outlined in [22]. In short, after releasing the cloud from the optical trap the binding energy of fermion pairs was rapidly increased by ramping the magnetic field within $2 \mathrm{~ms}(200 \mu \mathrm{s})$ to $690 \mathrm{G}$, in the far wings of the resonance on the BEC side. Here, fermion pairs were stable throughout further expansion. After a total of $11 \mathrm{~ms}(14 \mathrm{~ms}$ ) of expansion (in the remaining magnetic saddle-point potential) an image of either state $|1\rangle$ or state $|2\rangle$ was taken. For the condensate fraction data, the magnetic field was suddenly switched to $800 \mathrm{G}$ right before imaging. At this field the molecules absorb the probe light with the same strength as free atoms. The images revealed the center-of-mass wavefunction of the pairs and, for rotating clouds, the eventual presence of vortices. For the condensate fraction experiment, the $200 \mu$ s fast ramp 
to the BEC-side immediately after release from the trap ensured that even large condensates separated well from the normal, uncondensed component. Since the ramp was fast compared to the radial trapping period, the size of the expanded condensate was mostly governed by the residual mean-field interaction at $690 \mathrm{G}$, where $a=1400 a_{0}$.

Fig. 1 shows profiles of the two spin states for various spin-mixtures, on the BEC- and on the BCS-side of the resonance. Starting with a pure Fermi sea in state $|1\rangle$ we see how gradually, for increasing numbers in the second spin state $|2\rangle$ first a normal (uncondensed) cloud of fermion pairs emerges, then a condensate peak appears within the normal cloud (see also Fig. [3a, b). As the condensate size increases, vortices appear in the rotating cloud. Naturally, the largest condensates with the largest number of vortices are obtained for an equal mixture. Clearly, superfluidity in the strongly interacting Fermi gas is not constrained to a narrow region around the perfectly balanced spin-mixture, but instead superfluid flow is observed for large asymmetries in the populations.

Fig. 2 summarizes our findings for rotating spin-mixtures. It displays the number of detected vortices vs the population imbalance between the two spin states. The vortex number measures qualitatively how deep the system is in the superfluid phase: The higher the non-superfluid fraction, the faster the condensate's rotation will damp given the nonvanishing anisotropy $\left(\frac{\omega_{x}-\omega_{y}}{\omega_{x}+\omega_{y}} \approx 1.5 \%\right)$ of our trap [22, 38, 39]. We therefore observe how gradually, for decreasing interaction strength on the BCS-side, the superfluid window shrinks in size around the optimal situation of equal populations.

A more detailed map of the superfluid regime as a function of interaction strength and also temperature (see Fig. (5) was obtained from a study of condensate fractions, which were determined from cloud profiles such as in Fig. [3 Throughout the whole crossover region, pair condensation occurred for a broad range of population imbalances, demonstrating again the stability of the superfluid around the resonance.

An intriguing property of the superfluid state with imbalanced populations is the clear depletion in the excess fermions of the majority component, see Fig. Bl. The profiles in Fig. 3 present the axially integrated density, hence the true depletion in the 3D density is even stronger. The condensate seems to repel the excess fermions. This feature was observed after expansion at $690 \mathrm{G}$, where interactions are still strong (initially $1 / k_{F} a=$ 2.0). The expansion, at least in the region around the condensate, is hydrodynamic and should proceed as a scaling transformation [40, 41, 42]. Therefore, the depletion observed 
in ballistic expansion hints at spatial phase separation of the superfluid from the normal state. This effect was observed throughout the resonance region, and on resonance even when no magnetic field ramps were performed during expansion. However, to distinguish a phase separated state with equal densities in the superfluid region from more exotic states allowing unequal densities, a careful analysis of the three-dimensional density, reconstructed from the integrated optical densities, is necessary and will be the subject of a future study.

We did not observe (by simultaneously imaging along the long and short axis) a modulation in the condensate density as would be predicted for the FFLO state 27, 43, 44]. However, this state is predicted to be favored only in a narrow region of parameter space and might have escaped our attention.

The condensate fraction was determined from the minority component, which in all cases is very well fit by a gaussian for thermal molecules and unpaired atoms, plus a parabolic Thomas-Fermi-profile for the condensate. Fig. 圆 shows the condensate fraction obtained for varying population difference and temperature, and for magnetic fields or interaction strengths around resonance. The data for $754 \mathrm{G}$, on the BEC-side of the resonance, shows condensation over almost the entire range of population imbalance. As the interaction strength is increased towards resonance, the condensate fraction for equal mixtures grows [15]. However, for large population asymmetries it disappears. The condensation window shrinks further as we cross the resonance and move to the BCS-side (Fig. [4 $\mathrm{d}-\mathrm{f}$ ).

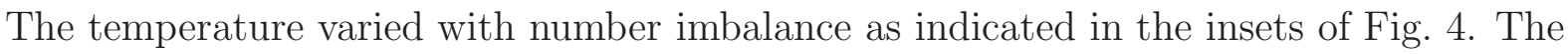
maximum for equal mixtures at $754 \mathrm{G}$ is likely due to the energy release when more molecules were formed. The observed critical population imbalance was only weakly dependent on temperature. This may reflect that the pairing gap is only a weak function of temperature, for temperatures well below the critical temperature for superfluidity [45]. The critical imbalance at our coldest temperatures will thus essentially coincide with its value at zero temperature.

On resonance, where the scattering length $a$ diverges, the system is in the unitary regime [46, 47, 48, 49, 50], where the only remaining energy scales of the system are the Fermi energies $E_{F, 1}$ and $E_{F, 2}$ of the two spin components. The breakdown of superfluidity will occur for a certain universal ratio of these two or equivalently, in a harmonic trap, for a certain critical population imbalance. We determine this universal number to be $\delta_{c} \approx \pm 70(3) \%$ for our approximately harmonic trapping potential. This corresponds to a Fermi energy 
difference $\delta E_{F}=E_{F, 2}-E_{F, 1}=\left(\left(1+\delta_{c}\right)^{1 / 3}-\left(1-\delta_{c}\right)^{1 / 3}\right) E_{F}=0.53(3) E_{F}$, with $E_{F}$ the Fermi energy of an equal mixture of non-interacting fermions. The standard BCS state is predicted [1] to break down for a critical chemical potential difference $\delta \mu=\sqrt{2} \Delta$. On resonance, however, Monte-Carlo studies predict [26] the superfluid breakdown to occur when $\delta \mu=2.0(1) \Delta=1.0(1) E_{F}$. Only in the weakly interacting regime do the chemical potentials equal the Fermi energies. Quantitative agreement with the Monte-Carlo study would require that $\delta \mu \approx 2 \delta E_{F}$. This is not unreasonable given the fact that interactions will reduce the chemical potential of the minority component. In a preliminary analysis, we indeed find close agreement with theory.

Fig. 5 summarizes our findings. It shows the critical mismatch in Fermi energies for which we observed the breakdown of superfluidity as well as the pairing gap $\Delta$ versus the interaction parameter $1 / k_{F} a$. Far on the BEC-side of the resonance the superfluid is very robust with respect to population imbalance. Here, pairing is dominantly a two-body process: The smallest cloud of atoms in state $|1\rangle$ will fully pair with majority atoms in state $|2\rangle$ and condense at sufficiently low temperatures. On the BCS-side of the resonance, however, pairing is purely a many-body effect and depends on the density of the two Fermi clouds. As the density of the minority component becomes smaller, the net energy gain from forming a pair condensate will decrease. Even at zero temperature, this eventually leads to the breakdown of superfluidity and the quantum phase transition to the normal state. We have experimentally confirmed the qualitative picture that fermionic superfluidity breaks down when the difference in chemical potentials between the two species becomes larger than the pairing gap.

In conclusion, we have observed superfluidity with imbalanced spin populations. Contrary to expectations for the weakly interacting case, superfluidity in the resonant region is extremely stable versus population imbalance. As the asymmetry is increased, we observe the quantum phase transition to the normal state, known as the Pauli limit of superfluidity. Our observation opens up intriguing possibilities for further studies on mismatched Fermi surfaces. One important aspect concerns the density distribution in the superfluid regime. Standard BCS theory allows only equal spin densities, which would entail complete phase separation of the superfluid from the normal density. More exotic solutions [6] allow superfluidity also with imbalanced densities. Equally fascinating is the nature of the strongly correlated normal state slightly below resonance. For sufficient 
population imbalance we have the remarkable situation that bosonic molecules, stable even in isolation, do not condense at zero temperature, due to the presence of the Fermi sea.

\section{References and Notes}

* to whom correspondence should be addressed. E-mail Zwierlei@mit.edu

[1] A. M. Clogston, Phys. Rev. Lett. 9, 266 (1962).

[2] P. Fulde and R. A. Ferrell, Phys. Rev. 135, A550 (1964).

[3] A. J. Larkin and Y. N. Ovchinnikov, Zh. Eksp. Teor. Fiz. 47, 1136 (1964), [Sov. Phys. JETP 20, $762(1965)]$.

[4] G. Sarma, J. Phys. Chem. Solids 24, 1029 (1963).

[5] W. V. Liu and F. Wilczek, Phys. Rev. Lett. 90, 047002 (2003).

[6] R. Casalbuoni and G. Nardulli, Rev. Mod. Phys. 76, 263 (2004), and references therein.

[7] H. A. Radovan, N. A. Fortune, T. P. Murphy, S. T. Hannahs, E. C. Palm, S. W. Tozer, and D. Hall, Nature 425, 51 (2003).

[8] A. Bianchi, R. Movshovich, C. Capan, P. G. Pagliuso, and J. L. Sarrao, Phys. Rev. Lett. 91, 187004 (2003).

[9] D. Vollhardt and P. Wölfle, The Superfluid Phases of Helium 3 (Taylor \& Francis, London, 1990).

[10] C. A. Regal, C. Ticknor, J. L. Bohn, and D. S. Jin, Nature 424, 47 (2003).

[11] S. Jochim, M. Bartenstein, A. Altmeyer, G. Hendl, S. Riedl, C. Chin, J. H. Denschlag, and R. Grimm, Science 302, 2101 (2003).

[12] M. W. Zwierlein, C. A. Stan, C. H. Schunck, S. M. F. Raupach, S. Gupta, Z. Hadzibabic, and W. Ketterle, Phys. Rev. Lett. 91, 250401 (2003).

[13] M. Bartenstein, A. Altmeyer, S. Riedl, S. Jochim, C. Chin, J. H. Denschlag, and R. Grimm, Phys. Rev. Lett. 92, 120401 (2004).

[14] C. A. Regal, M. Greiner, and D. S. Jin, Phys. Rev. Lett. 92, 040403 (2004).

[15] M. W. Zwierlein, C. A. Stan, C. H. Schunck, S. M. F. Raupach, A. J. Kerman, and W. Ketterle, Phys. Rev. Lett. 92, 120403 (2004).

[16] T. Bourdel, L. Khaykovich, J. Cubizolles, J. Zhang, F. Chevy, M. Teichmann, L. Tarruell, 
S. J. J. M. F. Kokkelmans, and C. Salomon, Phys. Rev. Lett. 93, 050401 (2004).

[17] J. Kinast, S. L. Hemmer, M. E. Gehm, A. Turlapov, and J. E. Thomas, Phys. Rev. Lett. 92, $150402(2004)$.

[18] M. Bartenstein, A. Altmeyer, S. Riedl, S. Jochim, C. Chin, J. H. Denschlag, and R. Grimm, Phys. Rev. Lett. 92, 203201 (2004).

[19] C. Chin, M. Bartenstein, A. Altmeyer, S. Riedl, S. Jochim, J. H. Denschlag, and R. Grimm, Science 305, 1128 (2004).

[20] J. Kinast, A. Turlapov, J. E. Thomas, Q. Chen, J. Stajic, and K. Levin, Science 307, 1296 (2005).

[21] G. B. Partridge, K. E. Strecker, R. I. Kamar, M. W. Jack, and R. G. Hulet, Phys. Rev. Lett. 95, 020404 (2005).

[22] M. W. Zwierlein, J. R. Abo-Shaeer, A. Schirotzek, C. H. Schunck, and W. Ketterle, Nature 435, 1047 (2005).

[23] D. M. Eagles, Phys. Rev. 186, 456 (1969).

[24] A. J. Leggett, in Modern Trends in the Theory of Condensed Matter. Proceedings of the XVIth Karpacz Winter School of Theoretical Physics, Karpacz, Poland, 1980, (SpringerVerlag, Berlin, Karpacz, Poland, 1980), pp. 13-27.

[25] P. Nozières and S. Schmitt-Rink, J. Low Temp. Phys. 59, 195 (1985).

[26] J. Carlson and S. Reddy, Phys. Rev. Lett. 95 (2005).

[27] A. Sedrakian, J. Mur-Petit, A. Polls, and H. Müther, Phys. Rev. A 72, 013613 (2005).

[28] C.-H. Pao, S.-T. Wu, and S.-K. Yip, preprint cond-mat/0506437

[29] D. E. Sheehy and L. Radzihovsky, preprint cond-mat/0508430

[30] Exotic superfluid states [6], most notably the FFLO-phase 2, 3] of non-zero momentum fermion pairs, are predicted to be more stable configurations than typical BCS superfluidity, but eventually also these will be destroyed and the gas will turn normal.

[31] D. T. Son and M. A. Stephanov, preprint cond-mat/0507586.

[32] K. Yang, preprint cond-mat/0508484.

[33] L. Viverit, C. J. Pethick, and H. Smith, Phys. Rev. A 61, 053605 (2000).

[34] S. Powell, S. Sachdev, and H. P. Büchler, Phys. Rev. B 72, 024534 (2005).

[35] P. F. Bedaque, H. Caldas, and G. Rupak, Phys. Rev. Lett. 91, 247002 (2003).

[36] Z. Hadzibabic, S. Gupta, C. A. Stan, C. H. Schunck, M. W. Zwierlein, K. Dieckmann, and 
W. Ketterle, Phys. Rev. Lett. 91, 160401 (2003).

[37] M. Bartenstein, A. Altmeyer, S. Riedl, R. Geursen, S. Jochim, C. Chin, J. H. Denschlag, R. Grimm, A. Simoni, E. Tiesinga, et al., Phys. Rev. Lett. 94, 103201 (2004).

[38] J. R. Abo-Shaeer, C. Raman, and W. Ketterle, Phys. Rev. Lett. 88, 070409 (2002).

[39] D. Guéry-Odelin, Phys. Rev. A 62, 033607 (2000).

[40] Y. Kagan, E. L. Surkov, and G. V. Shlyapnikov, Phys. Rev. A 55, R18 (1997).

[41] C. Menotti, P. Pedri, and S. Stringari, Phys. Rev. Lett. 89, 250402 (2002).

[42] Y. Castin, Comptes Rendus Physique 5, 407 (2004).

[43] T. Mizushima, K. Machida, and M. Ichioka, Phys. Rev. Lett. 94, 060404 (2005).

[44] P. Castorina, M. Grasso, M. Oertel, M. Urban, and D. Zappal, Phys. Rev. A 72, 025601 (2005).

[45] A. A. Abrikosov, L. P. Gorkov, and I. E. Dzyaloshinski, Methods of Quantum Field Theory in Statistical Physics (Dover Publications, New York, 1975).

[46] G. Bertsch, INT Workshop on Effective Field Theory in Nuclear Physics (Seattle, February 1999).

[47] H. Heiselberg, Phys. Rev. A 63, 043606 (2001).

[48] T.-L. Ho, Phys. Rev. Lett. 92, 090402 (2004).

[49] T. D. Cohen, Phys. Rev. Lett. 95, 120403 (2005).

[50] K. M. O’Hara, S. L. Hemmer, M. E. Gehm, S. R. Granade, and J. E. Thomas, Science 298, 2179 (2002).

[51] We would like to thank Gretchen Campbell for a critical reading of the manuscript and XiaoGang Wen, Eugene Demler and Subir Sachdev for stimulating discussions. This work was supported by the NSF, ONR, ARO, and NASA. 


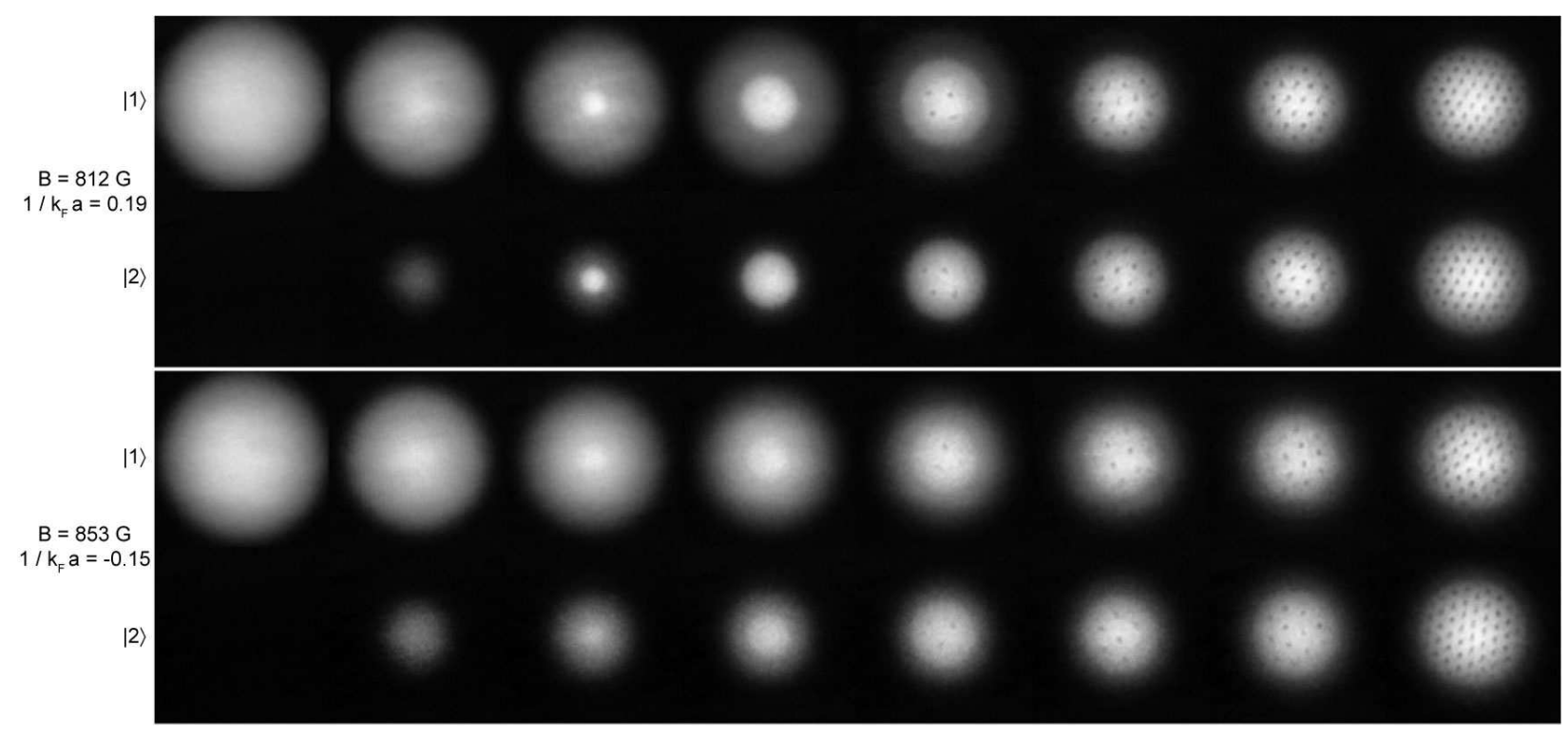

FIG. 1: Superfluidity in a strongly interacting Fermi gas with imbalanced populations. The upper (lower) pair of rows shows clouds prepared at $812 \mathrm{G}(853 \mathrm{G})$, where $1 / k_{F} a=0.2\left(1 / k_{F} a=-0.15\right)$. In each pair of rows, the upper picture shows state $|1\rangle$, the lower one state $|2\rangle$. For the $812 \mathrm{G}$ data, the population imbalance $\delta=\frac{N_{2}-N_{1}}{N_{1}+N_{2}}$ between $N_{1}$ atoms in state $|1\rangle$ and $N_{2}$ in state $|2\rangle$ was (from left to right) $100 \%, 90 \%, 80 \%, 62 \%, 28 \%, 18 \%, 10 \%$ and $0 \%$. For the $853 \mathrm{G}$ data, the mismatch was $100 \%, 74 \%, 58 \%, 48 \%, 32 \%, 16 \%, 7 \%$ and $0 \%$. For different $\delta$, the total number of atoms varied only within $20 \%$ around $N=7 \cdot 10^{6}$, with the exception of the endpoints $\delta=100 \%$ $\left(N=1 \cdot 10^{7}\right)$ and $\delta=0 \%\left(N=1.2 \cdot 10^{7}\right)$. The field of view of each image was $1.4 \mathrm{~mm} \times 1.4 \mathrm{~mm}$. 


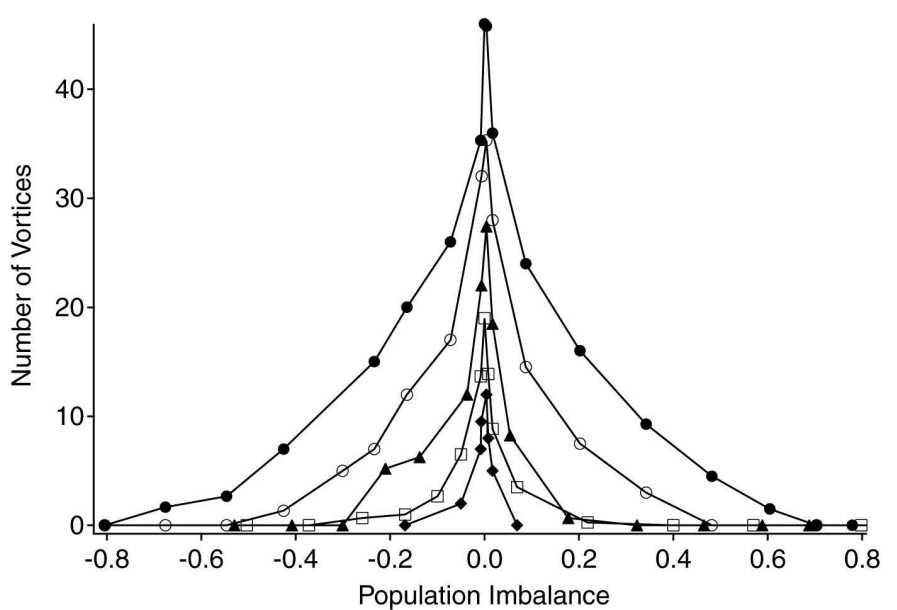

FIG. 2: Vortex number vs population imbalance for different interaction strengths. Results are shown for $812 \mathrm{G}$ or $1 / k_{F} a=0.2$ (filled circles), $853 \mathrm{G}\left(1 / k_{F} a=-0.15\right.$, empty circles), $874 \mathrm{G}$ $\left(1 / k_{F} a=-0.3\right.$, filled triangles), $896 \mathrm{G}\left(1 / k_{F} a=-0.4\right.$, empty squares) and $917 \mathrm{G}\left(1 / k_{F} a=-0.5\right.$, diamonds). 

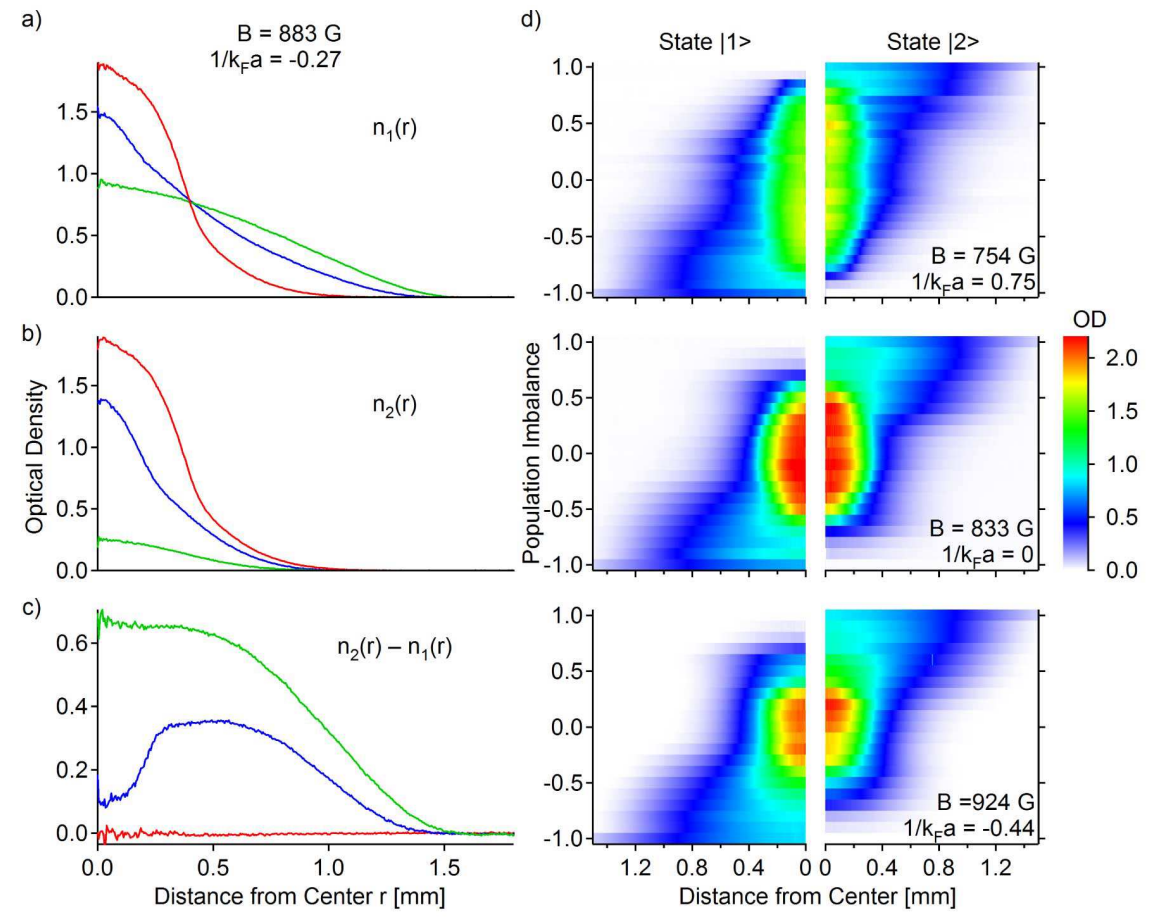

FIG. 3: Radial density profiles of the two components of a strongly interacting Fermi gas mixture with unequal populations. a) and b) Profiles of the component in state $|1\rangle$ and $|2\rangle$ resp., originating from $883 \mathrm{G}\left(1 / k_{F} a=-0.27\right)$. The imaging procedure is detailed in the text. The population imbalance was $\delta=0 \%$ (red), $\delta=46 \%$ (blue) and $\delta=86 \%$ (green). c) Difference between the distributions in state $|1\rangle$ and $|2\rangle$. The total number of atoms was $N=2.3 \cdot 10^{7}$. The clear dip in the blue curve caused by the pair condensate gives an indication for phase separation of the superfluid from the normal gas. d) Color-coded profiles of clouds prepared at three different interaction strengths. The profiles are azimuthal averages of the axially integrated density. The condensate is clearly visible as the dense central part surrounded by unpaired fermions or uncondensed molecules. 


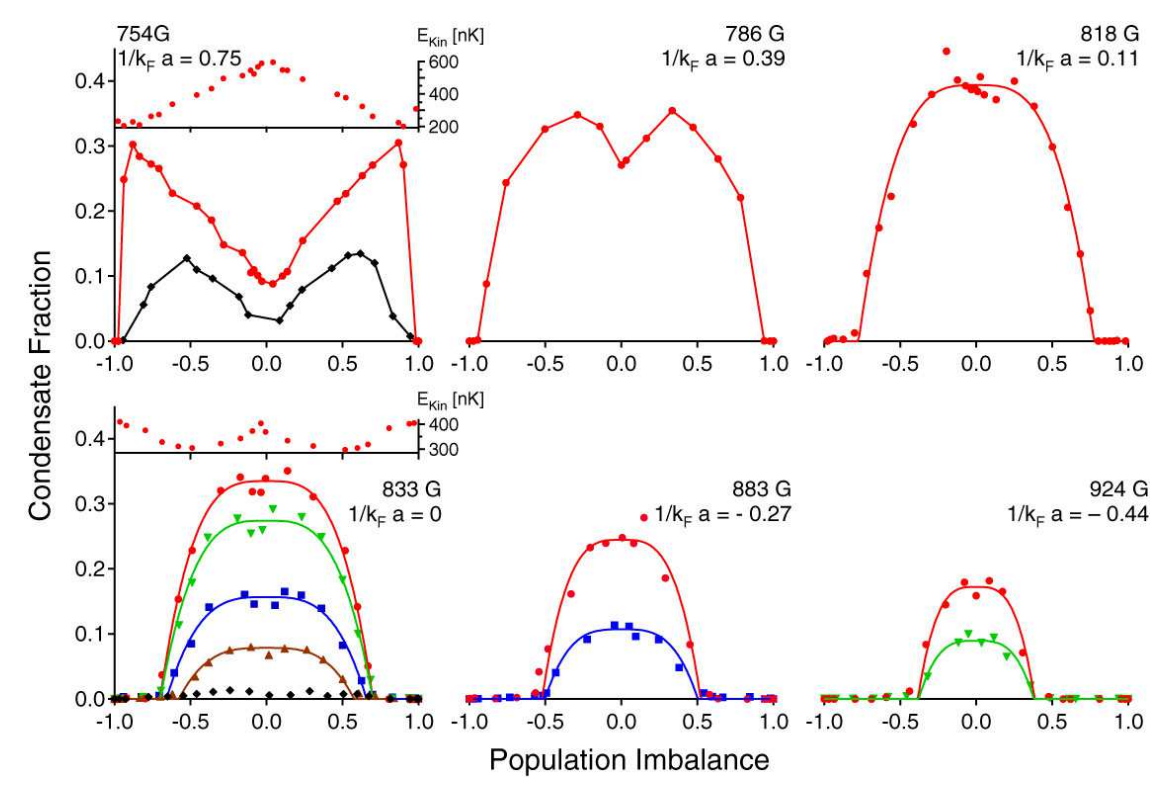

FIG. 4: Condensate fraction vs population imbalance for several temperatures and interaction strengths. The total number of atoms $N=2.3 \cdot 10^{7}$ is constant to within $20 \%$ for all data-points ( $T_{F}=1.9 \mu K$ for an equal mixture). For a given population imbalance, the uppermost curves for different magnetic fields are approximately isentropically connected. The different symbols correspond to different evaporation ramps. The average radial kinetic energy per molecule of thermal clouds in the minority component serves as an indicator for temperature and is shown in the insets for $754 \mathrm{G}$ and $833 \mathrm{G}$ for the coldest data. On resonance, for a population asymmetry of $50 \%$, we measure an energy of $k_{B} \cdot 300 \mathrm{nK}$ (circles), $345 \mathrm{nK}$ (inverted triangles), $390 \mathrm{nK}$ (squares), $420 \mathrm{nK}$ (triangles) and $505 \mathrm{nK}$ (diamonds). The critical population imbalance $\delta_{c}$ for the breakdown of condensation at $754 \mathrm{G}$ is about $\delta_{c}^{754} \approx 96 \%$ and at $786 \mathrm{G}$ it is $\delta_{c}^{786} \approx 95 \%$. For the data at higher magnetic fields we determine $\delta_{c}$ through a threshold fit to the first three data points with non-zero condensate fraction for each sign of asymmetry. Although we could have used any reasonable threshold function, empirically, it was found that the function $n_{c}\left(1-\left|\delta / \delta_{c}\right|^{3.3}\right)\left(n_{c}-\right.$ maximum condensate fraction) provided a good fit to all data points. Therefore it was used for the threshold fits and is shown as a guide to the eye. 


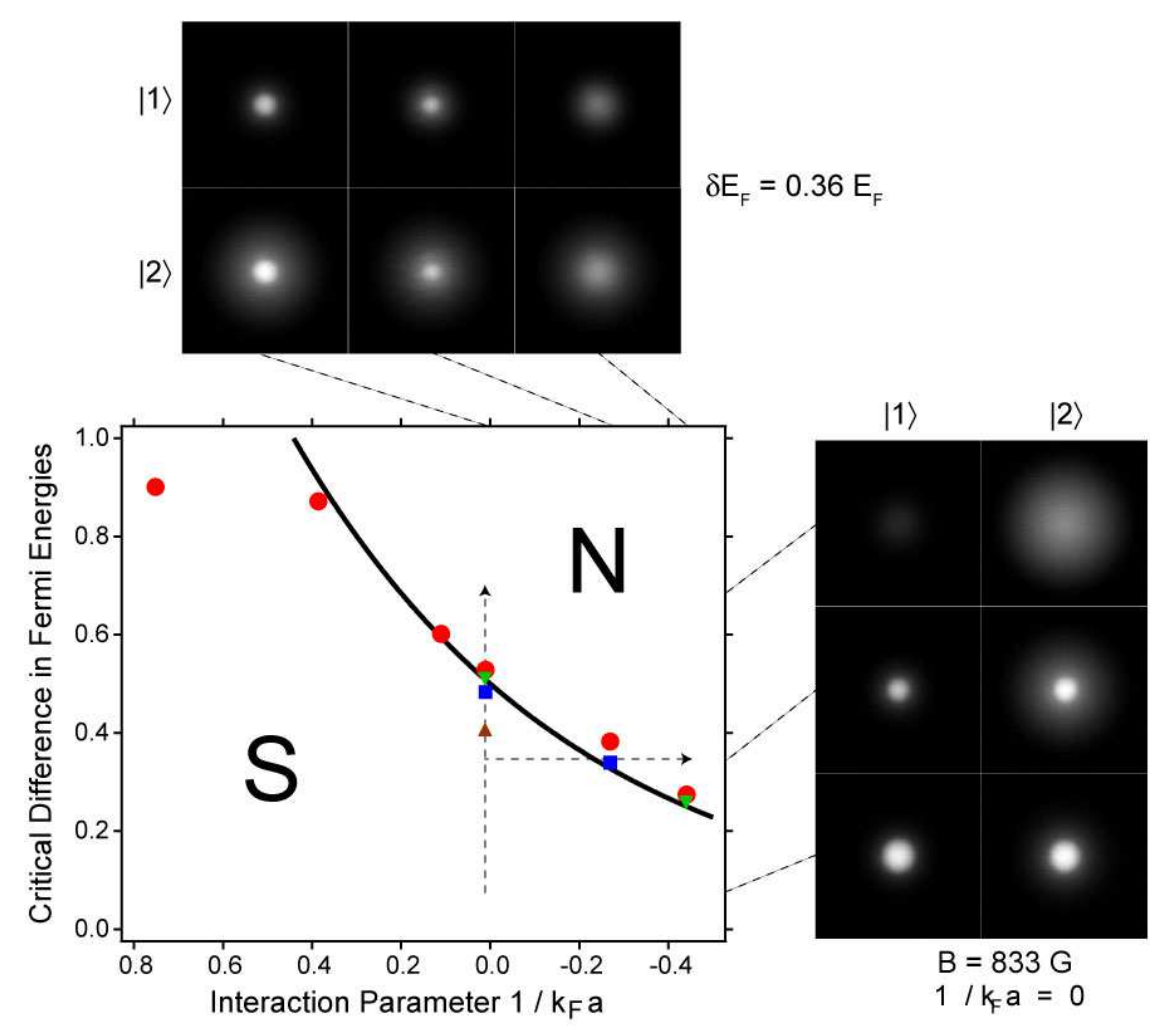

FIG. 5: Critical difference in Fermi energies $\delta E_{F}$ between the two spin states for which the superfluid to normal transition is observed. $\delta E_{F}$ for each interaction strength and temperature is obtained from the critical population imbalance determined in Fig. 目 using $\delta E_{F} / E_{F}=$ $\left(1+\delta_{c}\right)^{1 / 3}-\left(1-\delta_{c}\right)^{1 / 3}$. The symbols are defined in Fig. 4 . The line shows the expected variation of the pairing gap $\Delta$, where the value on resonance has been taken from 26] and the exponential behavior in the BCS-regime, $\Delta \sim e^{-\pi / 2 k_{F}|a|}$ was assumed. While the trend of $\delta E_{F}$ is expected to follow that of $\Delta$, the close agreement is coincidental. Representative density profiles illustrate the quantum phase transition for fixed interaction and for fixed population imbalance along the dashed lines. 